\title{
Sulfur cathode based on layered carbon matrix for high-performance Li-S batteries
}

Feng Wu ${ }^{\mathrm{a}, \mathrm{b}}$, Ji Qian ${ }^{\mathrm{a}}$, Renjie Chen ${ }^{\mathrm{a}, \mathrm{b},{ }^{*}}$, Teng Zhao ${ }^{\mathrm{a}, 1}$, Rui Xuc, Yusheng Ye ${ }^{\mathrm{a}}$, Wenhui Li ${ }^{\mathrm{a}}, \mathrm{Li} \mathrm{Li}^{\mathrm{a}, \mathrm{b}}$, Jun $\mathrm{Lu}^{\mathrm{c}, *}$, Khalil Amine ${ }^{\mathrm{c} *}$

${ }^{a}$ Beijing Key Laboratory of Environmental Science and Engineering, School of Chemical Engineering and Environment, Beijing Institute of Technology, Beijing 100081, PR China

${ }^{\mathrm{b}}$ National Development Center of High Technology Green Materials, Beijing 100081, PR China

${ }^{c}$ Chemical Sciences and Engineering Division, Argonne National Laboratory, 9700 South Cass Avenue, Lemont, IL 60439, USA

*Corresponding Author. E-mail address: chenrj@bit.edu.cn (R. J. Chen); junlu@anl.gov (J. Lu); and amine@anl.gov (K. Amine)

${ }^{1}$ Teng Zhao now is a Ph. D. candidate at University of Cambridge, UK 


\begin{abstract}
A novel carbon/sulfur composite has been fabricated by means of thermal and hydro-thermal treatments to serve as the cathode in Li-S batteries. The carbon matrix consists of graphene nanosheet (GS) and multiwalled carbon nanotube (MWCNT). The "GS/MWCNT@S" composite allows for infiltration of electrolyte into the cathode, assists in entrapment of polysulfide intermediates, and accommodates some of the stress and volume expansion that occurs during charge-discharge processes. In addition, the uniform distribution of sulfur in the conductive carbon matrix promotes utilization of the active materials. A Li-S cell containing the GS/MWCNT@S cathode delivered a capacity of 1290.8 mAh/g and exhibited stable specific capacities up to $612.1 \mathrm{mAh} / \mathrm{g}$ after 200 cycles at $0.1 \mathrm{C}$. These results demonstrate that this cathode material is a promising candidate for rechargeable lithium batteries with high energy density.
\end{abstract}

KEYWORDS: lithium-sulfur battery, cathode, graphene sheet, multi-walled carbon nanotube, carbon matrix 


\section{Introduction}

Combustion reactions are responsible for the global economy rapidly expanding over the last two centuries, but this expansion has resulted in energy sustainability and environmental problems. One solution for overcoming these problems is developing more advanced batteries. [1] Because of their high specific energies and zero emissions, Li-containing batteries are thus becoming increasingly the focus of research attention. [2-4] Lithium-sulfur batteries are especially promising electrochemical systems because of their high theoretical capacity of 1672 $\mathrm{mAh} / \mathrm{g}$ and energy density of $2600 \mathrm{Wh} / \mathrm{kg}$. [5] Also, the active material is sulfur, which is economical, abundant, and nontoxic. However, the mass commercialization of Li-S batteries has been impeded by several obstacles. [2,3,6,7] First, the poor conductivity of sulfur and the corresponding discharge products $\left(\mathrm{Li}_{2} \mathrm{~S}_{2} / \mathrm{Li}_{2} \mathrm{~S}\right)$ results in unstable electrochemical contact within sulfur electrodes. Second, soluble polysulfide intermediates are generated during charge-discharge processes in Li-S cells, and these intermediates result in a shuttle mechanism that decreases use of the active material and, thus, the coulombic efficiency. Third, the high reactivity of lithium anodes with components of the electrolyte (e.g., polysulfides and solvents) leads to capacity fading and, potentially, to thermal runway

Sulfur composites with well-designed structures have been developed to overcome these problems. Most such efforts have focused on developing carbon-based sulfur cathodes. Carbon materials generally exhibit high conductivity and favorable adsorption properties, and are mechanically robust. Carbon-based materials with different pore structures (macro, meso, or micro) have been investigated. Nazar et al. first used the mesoporous carbon material CMK-3 as a host for sulfur and achieved excellent electrochemical performance in the resulting cells. [8] Other mesoporous carbons have since been developed and have performed well in Li-S cells. [9-15] Microporous carbon is also commonly used in Li-S cells. [16-18] Hierarchical porous carbon can simultaneously exploit different pore sizes. [9,18-23] Various pore sizes have been tested to accommodate sulfur, transfer lithium ions, and buffer volume changes during cell operation. The pore size and pore volume greatly affect the cathode performance. Designing 
carbon matrices with specific morphologies is another strategy for tackling these problems. Carbon spheres, [24-26] fibers, [27,28] and tubes [29,30] have been used to improve the performance of sulfur cathodes.

Among various carbon materials, multi-walled carbon nanotubes (MWCNTs) and graphene sheets (GSs), which are one- and two-dimensional conductive carbons, respectively, have been used in the cathodes of Li-S cells. While MWCNT has been widely used as the conductive framework and surface absorbent in these cathodes, [31-34] it suffers from problems of low surface areas and poor ion transport. And while GS has high surface area, excellent conductivity, and good mechanical strength, [35-38] it suffers from restacking that may occur during charge-discharge processes.

For this effort, we hydrothermally prepared a nanostructured carbon matrix containing both MWCNTs and GSs, with sulfur being incorporated into the MWCNTs and GSs via two melt-diffusion treatments (see Figure 1a). This method results in sulfur being uniformly distributed throughout the carbon matrix, and the MWCNT/S and GS/S components show synergistic effects. The one-dimensional MWCNTs and two-dimensional GSs form a three-dimensional conductive network, and the MWCNTs within the composites can suppress the restacking of GSs. Our method resulted in a GS/MWCNT@S composite cathode with excellent electrochemical performance. The carbon matrix allows the electrolyte to access the active material and traps soluble polysulfides generated during charge-discharge processes.

\section{Results and discussion}

The detailed structure and composition of the GS/MWCNT@S composite were investigated by XRD and TGA. The XRD patterns of GSs, MWCNTs, sulfur, and the GS/MWCNT@S composite are shown in Figure 1(b). The GS/MWCNT@S composite exhibits a similar pattern to sulfur, indicating that sulfur in the composite is highly crystalline. 
Figure 1(c) shows the TGA curve of the GS/MWCNT@S composite, which indicates a 68 wt.\% sulfur loss at $150-250{ }^{\circ} \mathrm{C}$. The other weight losses, at $\sim 430$ and $530{ }^{\circ} \mathrm{C}$, are those of the GSs and MWCNTs, respectively. [39] These results reveal that GSs constitute $\sim 13$ wt.\% of the GS/MWCNT@S composite and 41wt.\% of the carbon matrix.

The morphology and structure of the GS/MWCNT@S composite and its components were characterized by SEM and TEM. Figure 2(a) shows an SEM image of the MWCNTs, and Figure 2(b) shows a TEM image of the GSs obtained by the hydrothermal treatment of GO. These two materials form the carbon matrix framework of the GS/MWCNT@S composite and allow for the incorporation of sulfur. An MWCNT@S-GS composite is formed before the second melt-diffusion treatment in Figure 1(a) and is an intermediate in the preparation of the GS/MWCNT@S composite. Figures 2(c) and (d) show SEM images of the MWCNT@S-GS and GS/MWCNT@S composites, respectively. They exhibit similar multi-layered morphologies, because of the alternate layering of GSs and MWCNTs. Thus, the structure prevents GS from restacking. It also provides open channels for access of electrolyte and buffers the sulfur volume changes during discharge-charge processes. The surface of the GS/MWCNT@S composite is smoother than that of the MWCNT@S-GS composite, because of the extra sulfur layer. The presence of the sulfur layer is confirmed in the EDX spectra inset in Figures 2(c) and (d). The GS/MWCNT@S and MWCNT@S-GS composite surfaces contain 71.83 and 8.56 wt.\% sulfur, respectively. Sulfur added through the second thermal treatment is predominantly anchored on GSs in the GS/MWCNT@S composite. This condition results in sulfur being uniformly distributed in the GS/MWCNT@S composite while raising the sulfur content in the composite. The uniform distribution of sulfur was confirmed by the EDX elemental maps of the GS/MWCNT@S composite in Figures 2(e) and (f). No bulk S particles are observed. High magnification SEM and TEM images of the GS/MWCNT@S composite are shown in Figures 2(g) and (h), respectively, which show the structure of the GS/MWCNT matrix. They indicate that the MWCNTs are attached to or encapsulated by GSs, and sulfur is anchored on the 
MWCNTs and GS. The interaction between the MWCNT/GS matrix and sulfur might contribute to effective anchoring of sulfur and trapping of soluble polysulfides.

Complementary information of the carbon matrix in the GS/MWCNT@S composite was provided by XPS and Raman spectroscopy. The XPS spectra of the C1s (284.5 eV) region of the GS/MWCNT@S composite are shown in Figure 3(a). Peaks in the C1s region are largely attributed to carbon in the GSs and indicate carbon in different forms (e.g., aromatic ring, C-C and C-OR bonding, and carbonyl). The GS/MWCNT@S composite was prepared from exfoliated GO, and the oxygen-containing functional groups of GS and GO are similar. The C1s peak intensities represent the relative amounts of the different carbon forms (Figure 3a, inset) and are significantly lower than those of GO. [40] This demonstrates that GO is significantly reduced during preparation of the GS/MWCNT@S composite.

The Raman spectrum of the GS/MWCNT@S composite is shown in Figure 3(b). The three main peaks are typical of the carbon matrix: $\mathrm{G}$ band at $\sim 1593 \mathrm{~cm}^{-1}$, D band at $\sim 1352 \mathrm{~cm}^{-1}$, and 2D band at $\sim 2704 \mathrm{~cm}^{-1}$. The $\mathrm{G}$ band is usually assigned to the E2g phonon of $\mathrm{sp}^{2}$-hybridized carbon, and the $\mathrm{D}$ band to defects or disorder in the carbon matrix. The intensity ratio of the $\mathrm{D}$ to G band $\left(\mathrm{I}_{\mathrm{D}} / \mathrm{I}_{\mathrm{G}}\right)$ of the GS/MWCNT@S composite is $\sim 0.93$. This ratio indicates that the aromatic structure is recovered by repairing defects, [41,42] which favor electron transport within the GS/MWCNT@S composite. The intensity ratio of the $2 \mathrm{D}$ to $\mathrm{G}$ band $\left(\mathrm{I}_{2 \mathrm{D}} / \mathrm{I}_{\mathrm{G}}\right)$ is 0.75 . An $\mathrm{I}_{2 \mathrm{D}} / \mathrm{I}_{\mathrm{G}}$ of $<1$ indicates multi-layered graphene within the composite. In addition, the large peak at $\sim 472$ $\mathrm{cm}^{-1}$ is characteristic of sulfur and is assigned to the A1 mode of the $\mathrm{S}-\mathrm{S}$ bond. [43]

The electrochemical properties of the GS/MWCNT@S composite were then investigated. Coin cells were assembled, with the composite and lithium foil acting as the cathode and anode, respectively. Figure 4(a) shows the CV curves of the GS/MWCNT@S cathode at 1.0-3.0 V and a scanning rate of $0.1 \mathrm{mV} / \mathrm{s}$. The $\mathrm{CV}$ curves are typical of those reported for sulfur, [44] because MWCNTs and GSs are electrochemically inactive within this potential range. The cathodic peaks at $\sim 2.3 \mathrm{~V}$ and $\sim 2.04 \mathrm{~V}$ correspond to the reduction of sulfur to lithium polysulfides, and 
further reduction to $\mathrm{Li}_{2} \mathrm{~S}_{2} / \mathrm{Li}_{2} \mathrm{~S}$, respectively. The anodic peaks overlap at $\sim 2.4 \mathrm{~V}$, because of the reverse conversion of $\mathrm{Li}_{2} \mathrm{~S}_{2} / \mathrm{Li}_{2} \mathrm{~S}$ to higher order polysulfides. [45]

The cycling performance of the GS/MWCNT@ $\mathrm{S}$ cathode at $0.1,0.2,0.5$, and $1 \mathrm{C}(1 \mathrm{C}=1675$ $\mathrm{mA} / \mathrm{g}_{\text {sulfur }}$ ) is shown in Figure $4(\mathrm{~b})$. Based on the sulfur loading, the cells deliver initial capacities of $1290.8,1205.9,1031.2$, and $790.8 \mathrm{mAh} / \mathrm{g}$, respectively. The difference in discharge capacities at different current rates decreases with increasing cycle number. This reflects rapid electronic/ionic transport and improved electrochemical properties of the GS/MWCNT@S composite.

Figure 4(c) shows charge-discharge curves of a Li-S cell containing the GS/MWCNT@S cathode during successive cycles at a current rate of $0.1 \mathrm{C}$. The discharge curves all contain plateaus at 2.3 and $2.1 \mathrm{~V}$ vs. $\mathrm{Li}^{+} / \mathrm{Li}$. This finding corresponds to the multistep reduction of sulfur to $\mathrm{Li}_{2} \mathrm{~S}_{2} / \mathrm{Li}_{2} \mathrm{~S}$, and is consistent with the CV curves shown in Figure 4(a). The curve shape remains constant between cycles, proving that the GS/MWCNT@S cathode is stable. The capacity is $612.1 \mathrm{mAh} / \mathrm{g}$ after 200 cycles.

A Li-S cell with an acetylene black/sulfur $(\mathrm{AB} / \mathrm{S})$ cathode of the same $\mathrm{S}$ loading was also tested for comparison. The cycling stability of the AB/S and GS/MWCNT@S cathodes at $0.1 \mathrm{C}$ is similar, as shown in Figure 4(d). The AB/S cathode predictably delivers a lower capacity, indicating that less active material is being used. The coulombic efficiency of the cell containing the AB/S cathode declines significantly after 20 cycles, while that for the GS/MWCNT@S cathode remains steady. These data suggest that a large amount of soluble polysulfides migrate from the $\mathrm{AB} / \mathrm{S}$ cathode into the electrolyte, resulting in shuttle reactions. This tendency appears to be overcome in the GS/MWCNT@S cathode, presumably because the composite structure inhibits dissolution of the polysulfides.

To verify that the GS/MWCNT matrix was responsible for the high cell performance, AC impedance measurements on GS/MWCNT@S and AB/S cathodes before cycling were performed, and the results are shown in Figure 5(a). The semicircular trend in data at high 
frequencies corresponds to the charge-transfer resistance $\left(\mathrm{R}_{\mathrm{ct}}\right)$, which is a measure of the charge transfer kinetics. The more linear trend in data at low frequencies is associated with the Warburg impedance of lithium ion diffusion within the cathode. A typical equivalent circuit model (Figure 5(a) inset) was used to analyze the Nyquist plots [46] and better understand the impedance spectra. The $\mathrm{R}_{\mathrm{ct}}$ values of the two cathodes are listed in the table inset in Figure 5(a). The $\mathrm{R}_{\mathrm{ct}}$ of the cell containing the GS/MWCNT@S cathode is $\sim 50 \%$ that for the AB/S cathode. This difference indicates that the GS/MWCNT@S composite can improve the electrochemical kinetics of sulfur in rechargeable lithium batteries. The carbon matrix in the GS/MWCNT@S cathode enhances electron transport and improves use of the active material. It also promotes infiltration of the organic electrolyte, high ion storage, and rapid ion diffusion upon prolonged cycling. The structure of the composite can potentially promote both ion and electron transport in Li-S batteries, as illustrated in Figure 5(b).

The ability of the carbon matrix to capture polysulfides in the GS/MWCNT@S composite was then investigated. The morphology of lithium anode surfaces cycled with the GS/MWCNT@S and $\mathrm{AB} / \mathrm{S}$ cathodes was compared after 50 cycles. As shown in Figures 6(a) and (b), respectively, the surface of the lithium anode cycled with the GS/MWCNT@S cathode is more smooth and compact than that cycled with the $\mathrm{AB} / \mathrm{S}$ cathode. The latter is covered by a deposition layer. The corresponding EDX spectra are shown in Figures 6(c) and (d). The detected sulfur contamination on the lithium anode implies the amount of $\mathrm{Li}_{2} \mathrm{~S}_{2} / \mathrm{Li}_{2} \mathrm{~S}$ and other sulfur-containing species and is much less for the GS/MWCNT@S cathode. The GS/MWCNT matrix can capture soluble polysulfides and can thus control the complicated parasitic reactions occurring on lithium metal. This capability alleviates anode corrosion, shuttle reactions, and the loss of active sulfur.

\section{Conclusions}

A GS/MWCNT@S composite was prepared by thermal and hydrothermal treatments. The GS/MWCNT matrix improves the electrical conductivity of the composite, allows for infiltration 
of electrolyte, assists the trapping of polysulfide intermediates, and accommodates stress and volume expansion during charge-discharge processes. In addition, the uniform sulfur distribution in the composite promotes use of the active material. When used as a cathode for a Li-S cell, the composite exhibits high specific capacity and good cycling stability. Optimizing the composite for use in rechargeable batteries remains the subject of future investigation.

\section{Experimental}

\section{Preparation of composite materials}

Preparation of the MWCNT@S-GS composite. MWCNT@S-GS composite was synthesized with the method described in our previous work. [47] GO was synthesized according to a modified Hummer's method. [48] Graphite powder was purchased from TianJin Fu Chen Chemical Reagents Factory (China). The MWCNT@S composite (containing 70\% sulfur by mass) was fabricated by using a typical melt-diffusion strategy. [33] Sulfur was purchased from Alfa Aesar (China), and MWCNTs from Shenzhen Nanotech Port Co. (China). $1 \mathrm{~g}$ of the MWCNT@S composite was dispersed in $100 \mathrm{~mL}$ of water/ethanol (1:1) and sonicated for 30 min. Then, $100 \mathrm{~mL}$ exfoliated GO solution $(\sim 3 \mathrm{mg} / \mathrm{mL})$ was added to above solution and sonicated for $1 \mathrm{~h}$. The obtained homogeneous black solution was sealed in a PTFE container and held at $95{ }^{\circ} \mathrm{C}$ in an oven for $24 \mathrm{~h}$. The product was collected by vacuum filtration and dried in a vacuum oven at $60{ }^{\circ} \mathrm{C}$ for $24 \mathrm{~h}$.

Preparation of the GS/MWCNT@S composite. Sulfur and the MWCNT@S-GS composite (7:13 by mass) were mixed by mechanical ball milling for $5 \mathrm{~h}$. The mixture was sealed in a PTFE container and heated to $155^{\circ} \mathrm{C}$ in an oven for $24 \mathrm{~h}$ under Ar.

Preparation of the acetylene black/sulfur composite. Sulfur and acetylene black (7:3 by mass) were mixed by mechanical ball milling, without thermal treatment.

\section{Characterization}


Measurements were recorded using a thermogravimetric/differential thermal analysis (TG/DTA) system (EXSTAR 6200, Japan) at a heating rate of $10{ }^{\circ} \mathrm{C} / \mathrm{min}$ in air. X-ray photoelectron spectroscopy (XPS) measurements were collected at room temperature by means of an electron spectroscopy for chemical analysis (ESCA) spectrometer (PHI-1600, USA) with a monochromatic Al- $K_{\alpha}(1486.6 \mathrm{eV})$ source. Raman spectra were measured using a spectrometer (Renishaw-1000, UK) with an excitation wavelength of $532 \mathrm{~nm}$. X-ray diffraction (XRD) patterns were recorded on a Rigaku X-ray diffractometer (Ultima IV-185, Japan) with Cu- $K_{\alpha}$ radiation $(\lambda=0.154 \mathrm{~nm})$. Particle morphologies were observed by field-emission scanning electron microscopy (SEM, FEI Quanta 250, USA) and high-resolution transmission electron microscopy (TEM, JEOL-2010, Japan). After cycling, cells were disassembled in a glove box. Electrodes were removed and thoroughly washed with excess 1,3-dioxolane (DOL, Alfa Aesar, China). The morphologies of the lithium anodes surfaces were studied by SEM (Hitachi S-4800, Japan). Elemental distributions were determined with an attached energy dispersive X-ray (EDX) apparatus.

\section{Electrochemical measurements}

The GS/MWCNT@S composite was formed into a cathode slurry. The composite (70\%), carbon black (20\%), and polyvinylidene fluoride (10\%) in N-methyl-2-pyrrolidinone were mixed, and then ball milled for $4 \mathrm{~h}$ to form a homogeneous slurry. After stirring, the slurry was coated on aluminum foil by a roll press. The coated electrodes were dried in a vacuum oven at $60{ }^{\circ} \mathrm{C}$ for 24 h. The electrodes were cut into discs of diameter $11 \mathrm{~mm}$. Two-electrode coin cells (CR2025) with Li foil counter electrodes were assembled in an Ar-filled glove box. The electrolyte was 1.0 mol/L bis(trifluoromethane)sulfonimide lithium (LiTFSI, 3M, USA) in dimethoxyethane (DME) and 1,3-dioxolane (DOL) solvents (1:1 v/v, Alfa Aesar, China). The cells were discharged and charged over the voltage range 1.0-3.0 V at different current densities. An electrochemical station (Wuhan LAND electronics, China) was used to test their cycling stability. Cyclic voltammetry (CV) curves were recorded on a CHI660c electrochemical workstation (Shanghai Chenhua, China) from 1.0 to $3.0 \mathrm{~V}$ to characterize the redox behavior and kinetic reversibility of 
the cells. Alternating current (AC) impedance was measured with a CHI660c electrochemical workstation. The AC amplitude was $\pm 5 \mathrm{mV}$, and the applied frequency range was $0.1 \mathrm{~Hz}$ to 100 $\mathrm{kHz}$.

\section{Acknowledgments}

This work was supported by the National Science Foundation of China (21373028); National 863 Program (2011AA11A256); New Century Educational Talents Plan of Chinese Education Ministry (NCET-12-0050); Beijing Novel Program (Z121103002512029); Ford University

Research Program (URP) project. This work was also supported by the U.S. Department of Energy under Contract DE-AC0206CH11357, with the main support provided by the Vehicle Technologies Office, Department of Energy (DOE) Office of Energy Efficiency and Renewable Energy (EERE).

\section{References}

[1] M. Armand, J. M. Tarascon, Nature 451 (2008) 652-657.

[2] S. Evers, L. F. Nazar, Accounts of Chemical Research 46 (2012) 1135-1143.

[3] A. Manthiram, Y. Fu, Y. S. Su, Accounts of Chemical Research 46 (2012) 1125-1134.

[4] P. G. Bruce, S. A. Freunberger, L. J. Hardwick, J. M. Tarascon, Nature Materials 11 (2012) 19-29.

[5] D. Peramunage, S. Licht, Science 261 (1993) 1029-1032.

[6] X. Ji, L. F. Nazar, Journal of Materials Chemistry 20 (2010) 9821-9826.

[7] S. S. Zhang, Journal of Power Sources 231 (2013) 153-162.

[8] X. Ji, K. T. Lee, L. F. Nazar, Nature Materials 8 (2009) 500-506.

[9] C. Liang, N. J. Dudney, J. Y. Howe, Chemistry of Materials 21 (2009) 4724-4730.

[10] J. Schuster, G. He, B. Mandlmeier, T. Yim, K. T. Lee, T. Bein, L. F. Nazar, Angewandte Chemie International Edition 51 (2012) 3591-3595. 
[11] J. Song, T. Xu, M. L. Gordin, P. Zhu, D. Lv, Y.-B. Jiang, Y. Chen, Y. Duan, D. Wang, Advanced Functional Materials 24 (2014) 1243-1250.

[12] M. Oschatz, S. Thieme, L. Borchardt, M. R. Lohe, T. Biemelt, J. Bruckner, H. Althues, S. Kaskel, Chemical Communications 49 (2013) 5832-5834.

[13] X. Huang, B. Sun, K. Li, S. Chen, G. Wang, Journal of Materials Chemistry A 1 (2013) 13484-13489.

[14] X. Liang, Z. Wen, Y. Liu, H. Zhang, L. Huang, J. Jin, Journal of Power Sources 196 (2011) 3655-3658.

[15] X. Tao, X. Chen, Y. Xia, H. Huang, Y. Gan, R. Wu, F. Chen, W. Zhang, Journal of Materials Chemistry A 1 (2013) 3295-3301.

[16] B. Zhang, X. Qin, G. R. Li, X. P. Gao, Energy \& Environmental Science 3 (2010) 1531-1537.

[17] S. Xin, L. Gu, N. H. Zhao, Y. X. Yin, L. J. Zhou, Y. G. Guo, L. J. Wan, Journal of the American Chemical Society 134 (2012) 18510-18513.

[18] D. W. Wang, G. M. Zhou, F. Li, K. H. Wu, G. Q. Lu, H. M. Cheng, I. R. Gentle, Physical Chemistry Chemical Physics 14 (2012) 8703-8710.

[19] G. Xu, B. Ding, P. Nie, L. Shen, H. Dou, X. Zhang, ACS Applied Materials \& Interfaces 6 (2014) 194-199.

[20] B. Ding, C. Z. Yuan, L. F. Shen, G. Y. Xu, P. Nie, X. G. Zhang, Chemistry - A European Journal 19 (2013) 1013-1019.

[21] G. He, X. Ji, L. Nazar, Energy \& Environmental Science 4 (2011) 2878-2883.

[22] S. Wei, H. Zhang, Y. Huang, W. Wang, Y. Xia, Z. Yu, Energy \& Environmental Science 4 (2011) 736-740.

[23] G. Xu, B. Ding, L. Shen, P. Nie, J. Han, X. Zhang, Journal of Materials Chemistry A 1 (2013) 4490-4496.

[24] N. Jayaprakash, J. Shen, S. S. Moganty, A. Corona, L. A. Archer, Angewandte Chemie International Edition 123 (2011) 6026-6030. 
[25] N. Brun, K. Sakaushi, L. Yu, L. Giebeler, J. Eckert, M. M. Titirici, Physical Chemistry Chemical Physics 15 (2013) 6080-6087.

[26] C. Zhang, H. B. Wu, C. Yuan, Z. Guo, X. W. Lou, Angewandte Chemie International Edition 51 (2012) 9592-9595.

[27] R. Elazari, G. Salitra, A. Garsuch, A. Panchenko, D. Aurbach, Advanced Materials 23 (2011) 5641-5644.

[28] G. Zheng, Y. Yang, J. J. Cha, S. S. Hong, Y. Cui, Nano Letters 11 (2011) 4462-4467.

[29] L. Wang, Y. Zhao, M. L. Thomas, H. R. Byon, Advanced Functional Materials 24 (2014) 2248-2252.

[30] J. Guo, Y. Xu, C. Wang, Nano Letters 11 (2011) 4288-4294.

[31] J. J. Chen, X. Jia, Q. J. She, C. Wang, Q. Zhang, M. S. Zheng, Q. F. Dong, Electrochimica Acta 55 (2010) 8062-8066.

[32] L. C. Yin, J. L. Wang, J. Yang, Y. N. Nuli, Journal of Materials Chemistry 21 (2011) 6807-6810.

[33] F. Wu, J. Chen, L. Li, T. Zhao, R. Chen, Journal of Physical Chemistry C 115 (2011) 24411-24417.

[34] J. J. Chen, Q. Zhang, Y. N. Shi, L. L. Qin, Y. Cao, M. S. Zheng, Q. F. Dong, Physical Chemistry Chemical Physics 14 (2012) 5376-5382.

[35] J. Z. Wang, L. Lu, M. Choucair, J. A. Stride, X. Xu, H. K. Liu, Journal of Power Sources 196 (2011) 7030-7034.

[36] H. Xu, Y. F. Deng, Z. C. Shi, Y. X. Qian, Y. Z. Meng, G. H. Chen, Journal of Materials Chemistry A 1 (2013) 15142-15149.

[37] J. Jin, Z. Y. Wen, G. Q. Ma, Y. Lu, Y. M. Cui, M. F. Wu, X. Liang, X. W. Wu, RSC Advances 3 (2013) 2558-2560.

[38] H. Wang, Y. Yang, Y. Liang, J. T. Robinson, Y. Li, A. Jackson, Y. Cui, H. Dai, Nano Letters 11 (2011) 2644-2647. 
[39] D. Wang, D. Choi, J. Li, Z. Yang, Z. Nie, R. Kou, D. Hu, C. Wang, L. V. Saraf, J. Zhang, I. A. Aksay, J. Liu, ACS Nano 3 (2009) 907-914.

[40] S. Stankovich, D. A. Dikin, R. D. Piner, K. A. Kohlhaas, A. Kleinhammes, Y. Jia, Y. Wu, S. T. Nguyen, R. S. Ruoff, Carbon 45 (2007) 1558-1565.

[41]K. Krishnamoorthy, M. Veerapandian, G.-S. Kim, S. Jae Kim, Current Nanoscience 8 (2012) 934-938.

[42] Y. Zhou, Q. Bao, L. A. L. Tang, Y. Zhong, K. P. Loh, Chemistry of Materials 21 (2009) 2950-2956.

[43] A. T. Ward, Journal of Physical Chemistry 72 (1968) 4133-4139.

[44] M. K. Song, E. J. Cairns, Y. Zhang, Nanoscale 5 (2013) 2186-2204.

[45] Y. V. Mikhaylik, J. R. Akridge, Journal of the Electrochemical Society 151 (2004) A1969-A1976.

[46] V. S. Kolosnitsyn, E. V. Kuzmina, E. V. Karaseva, S. E. Mochalov, Journal of Power Sources 196 (2011) 1478-1482.

[47] R. J. Chen, T. Zhao, J. Lu, F. Wu, L. Li, J. Z. Chen, G. Q. Tan, Y. S. Ye, K. Amine, Nano Letters 13 (2013) 4642-4649.

[48] J. P. Zhao, S. F. Pei, W. C. Ren, L. B. Gao, H. M. Cheng, ACS Nano 4 (2010) 5245-5252. 
(a)

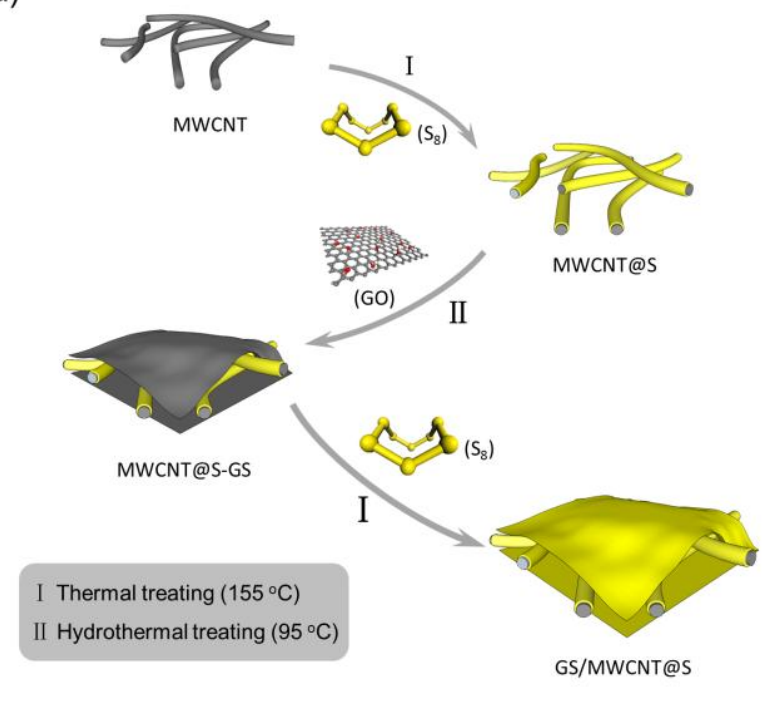

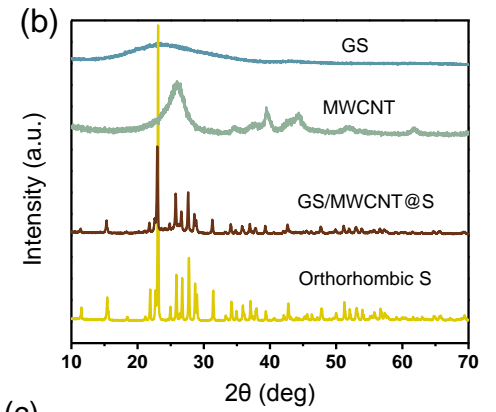

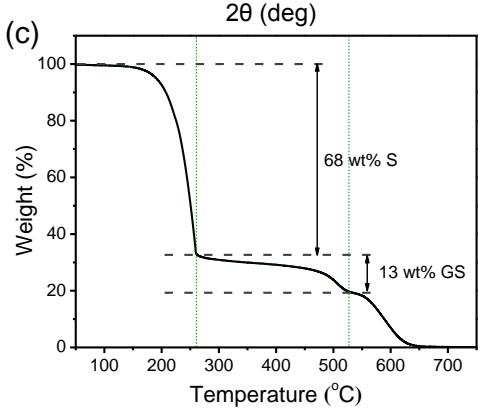

Figure 1. (a) Preparation of the GS/MWCNT@S composite. The yellow coating indicates sulfur distributed on MWCNTs and GSs. (b) XRD patterns of S, GSs, MWCNTs, and the GS/MWCNT@S composite. (c) TGA curves of the GS/MWCNT@S composite recorded in air at a heating rate of $10^{\circ} \mathrm{C} / \mathrm{min}$. 

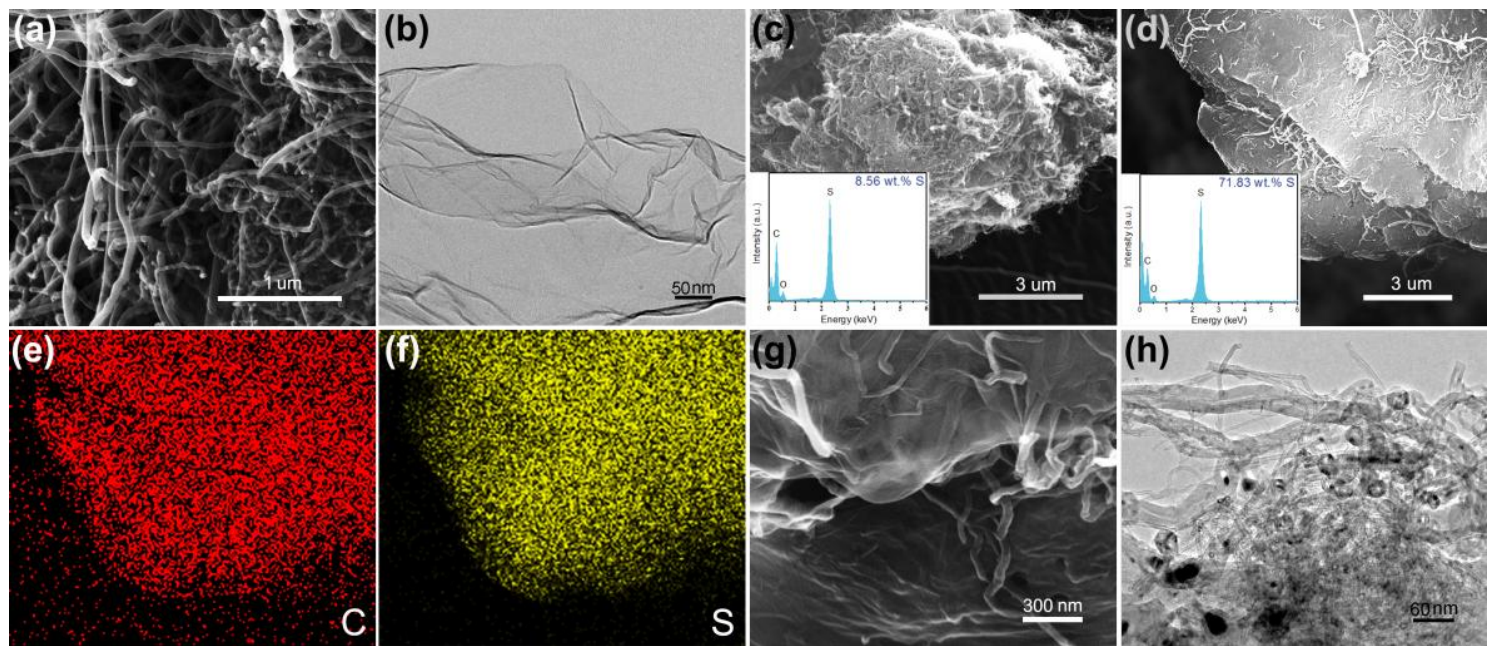

Figure 2. (a) SEM image of MWCNTs. (b) TEM image of GSs. SEM images of the (c) MWCNT@S-GS and (d) GS/MWCNT@S composites, with insets showing corresponding EDX spectra. EDX elemental maps of (e) carbon and (f) sulfur distributions in (d). High magnification (g) SEM and (h) TEM images of the GS/MWCNT@S composite. 

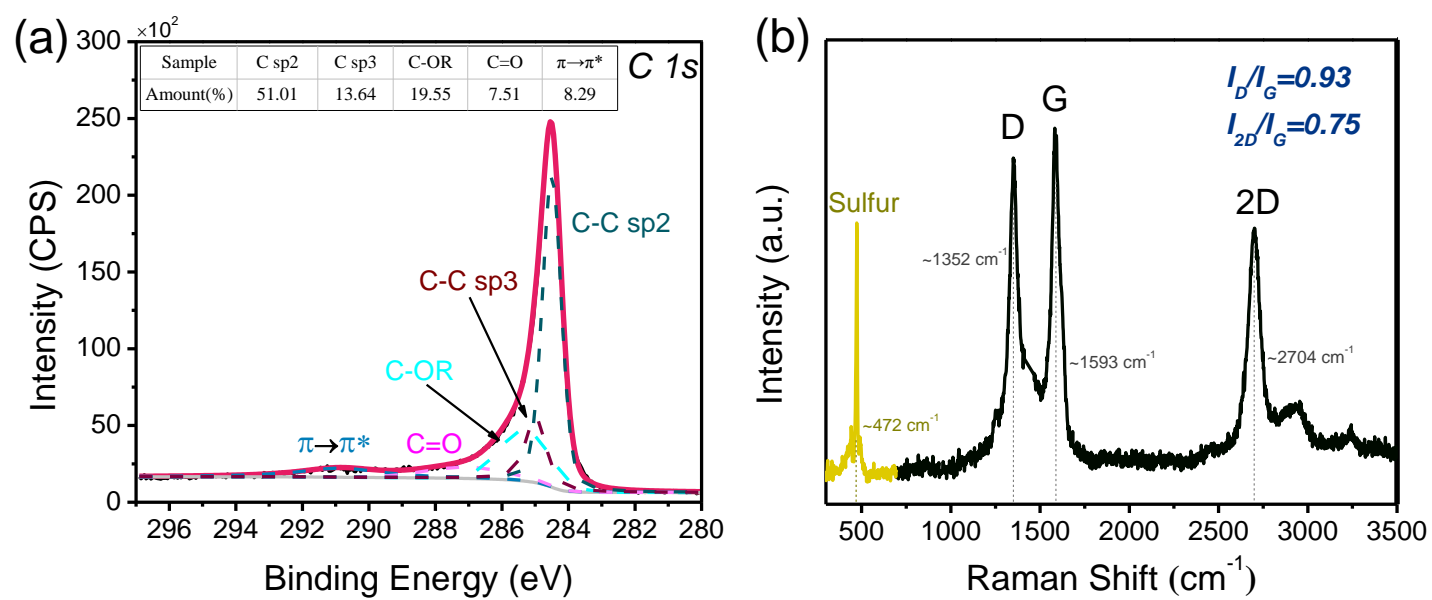

Figure 3. (a) XPS spectra of the C1s regions and (b) Raman spectrum of the GS/MWCNT@S composite. 

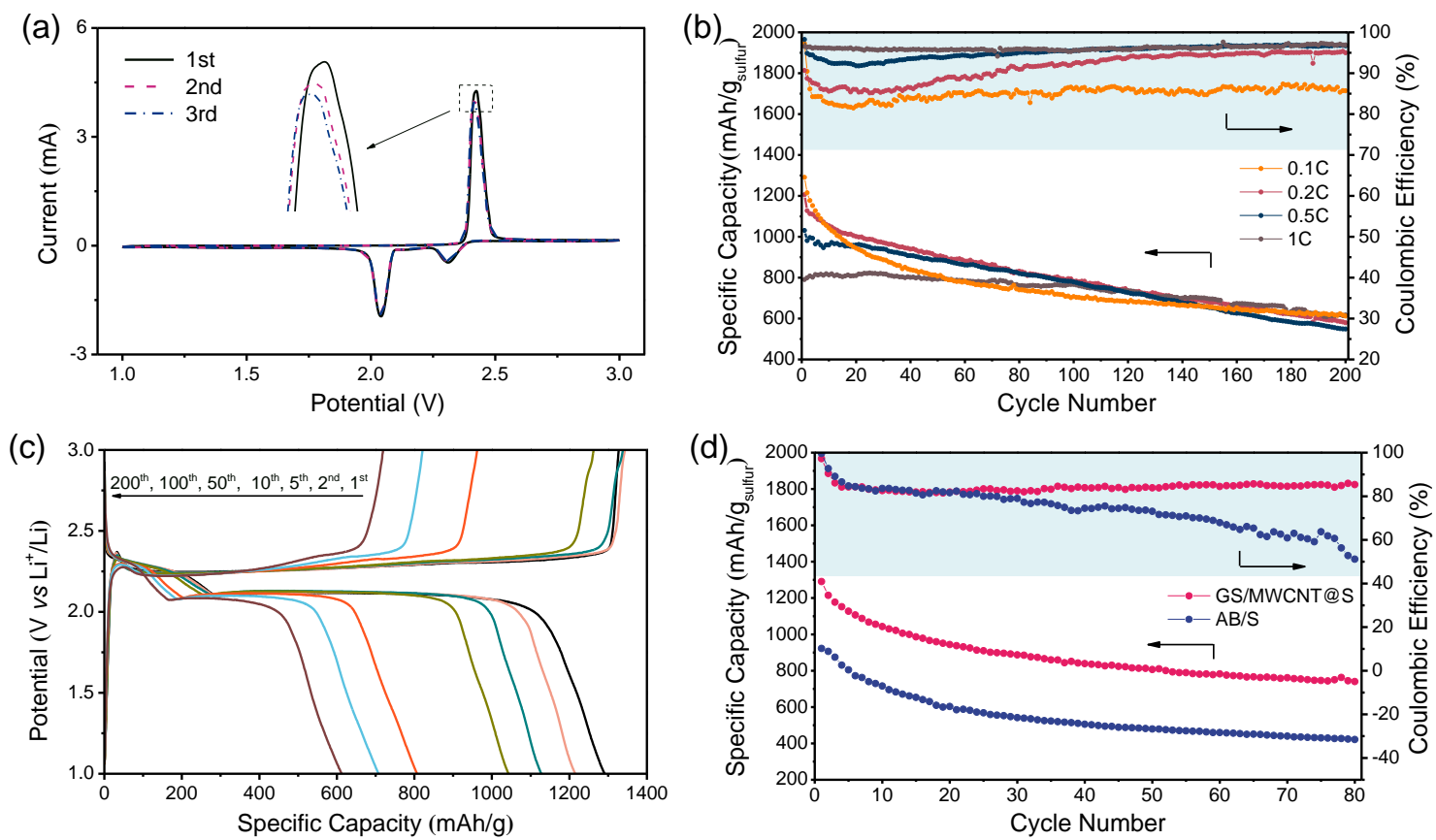

Figure 4. (a) CV curves of the GS/MWCNT@S cathode at a scanning rate of $0.1 \mathrm{mV} / \mathrm{s}$. (b) Cycling performance of the Li-S cells containing GS/MWCNT@S cathode at different current rates ( $\left.1 \mathrm{C}=1675 \mathrm{~mA} / \mathrm{g}_{\text {sulfur }}\right)$. (c) Discharge/charge voltage profiles of a $\mathrm{Li}-\mathrm{S}$ cell containing the GS/MWCNT@S cathode at a current rate of $0.1 \mathrm{C}$. (d) Cycling stability of cells containing GS/MWCNT@S and AB/S cathodes at a current rate of $0.1 \mathrm{C}$. 

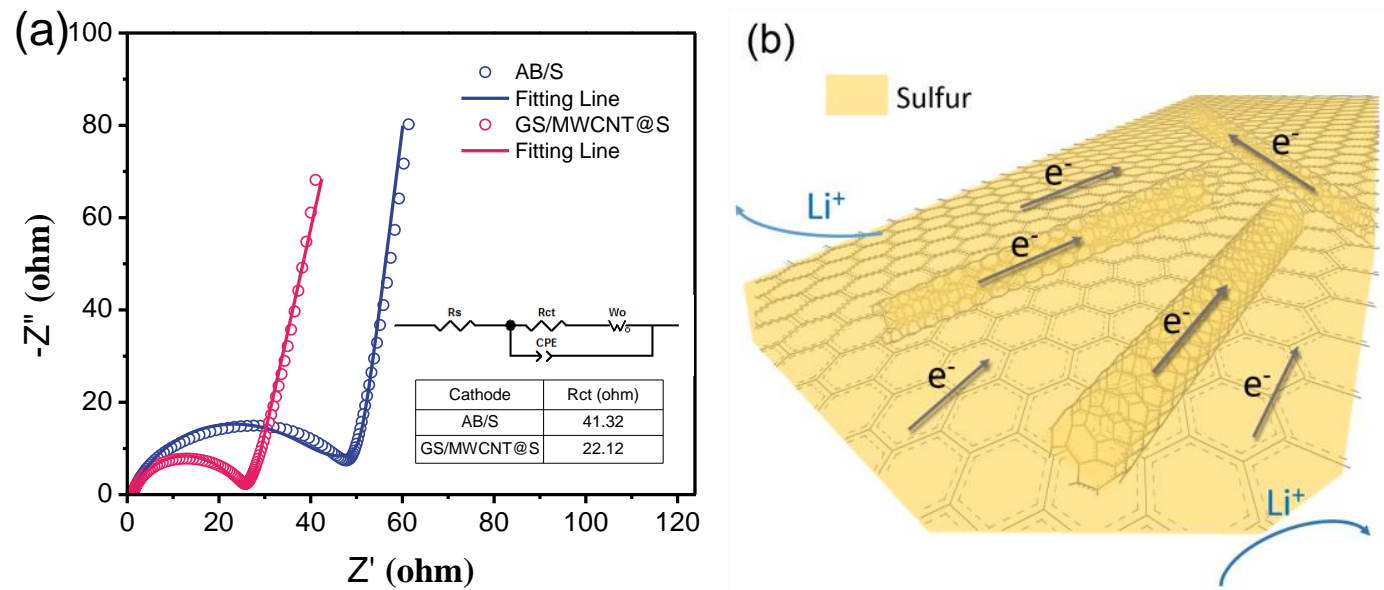

Figure 5. (a) $A C$ impedance measurements of cells containing $A B / S$ and GS/MWCNT@S composites before cycling. (b) Schematic showing the characteristics of the GS/MWCNT@S cathode in a Li-S cell. 

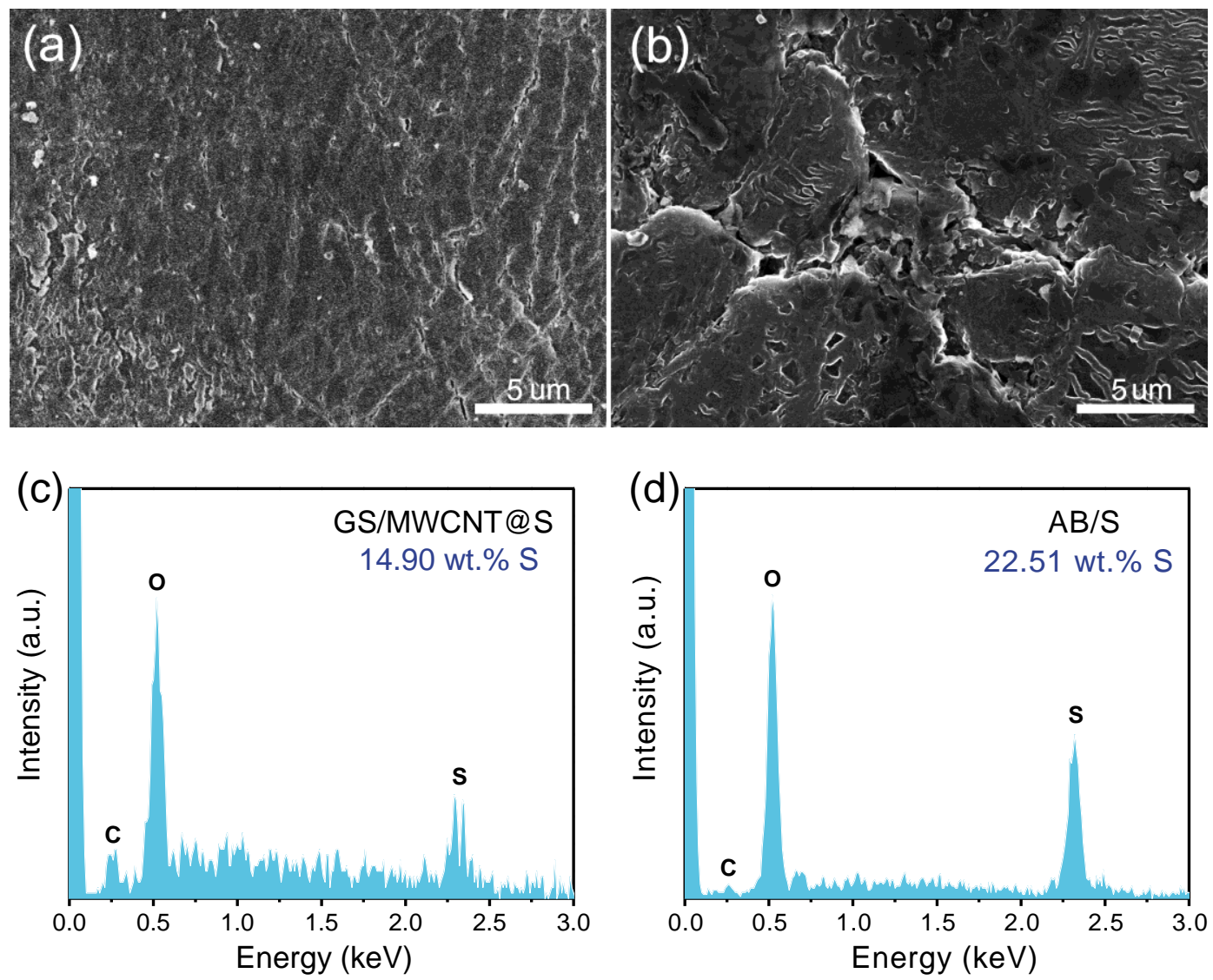

Figure 6. Morphology of lithium anode surfaces cycled with the (a) GS/MWCNT@S and (b) $A B / S$ cathodes, and (c, d) corresponding EDX spectra. All cells were disassembled after 50 cycles at $0.1 \mathrm{C}$ 


\section{Graphical Abstract}
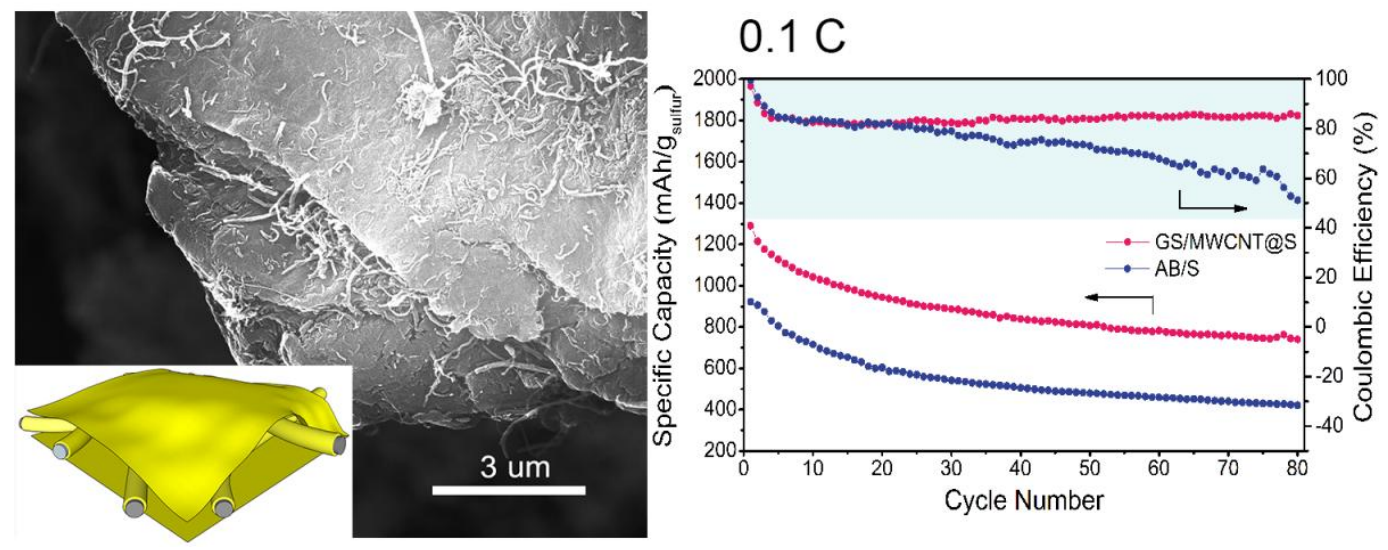